\title{
BMJ Open Lived experiences of social support in Paralympic swimmers: A protocol for a qualitative study
}

\author{
Beth Aitchison, ${ }^{1}$ Andrew Soundy (D) , ${ }^{1}$ Paul Martin, ${ }^{2}$ Alison Rushton (D) , ${ }^{3}$ \\ Nicola R Heneghan (D) ${ }^{3}$
}

To cite: Aitchison B, Soundy A, Martin P, et al. Lived experiences of social support in Paralympic swimmers: A protocol for a qualitative study. BMJ Open 2020;10:e039953. doi:10.1136/ bmjopen-2020-039953

- Prepublication history for this paper is available online. To view these files, please visit the journal online (http://dx.doi. org/10.1136/bmjopen-2020039953).

Received 30 April 2020 Revised 18 August 2020 Accepted 20 August 2020

Check for updates

(C) Author(s) (or their employer(s)) 2020. Re-use permitted under CC BY-NC. No commercial re-use. See rights and permissions. Published by BMJ.

${ }^{1}$ School of Sport, Exercise and Rehabilitation Sciences, University of Birmingham, Birmingham, UK

${ }^{2}$ The English Institute of Sport, London, UK

${ }^{3}$ Centre of Precision

Rehabilitation for Spinal Pain (CPR Spine), School of Sport, Exercise and Rehabiliation Sciences, University of Birmingham, Birmingham, UK

Correspondence to Dr Nicola R Heneghan; n.heneghan@bham.ac.uk

\section{ABSTRACT}

Introduction Over the past decade, there has been an increase in awareness of and investment into disability sport as a result of the 'Paralympic Movement'. The provision of personal and professional support to elite athletes is important for the well-being and success of the athlete, with various studies advocating a holistic approach to performance enhancement. However, little is known about social support experiences in elite para-swimming. Swimming is a popular Paralympic sport and the British para swimmers have been very successful in recent years, most recently winning 47 medals at Rio 2016. This study will be the first to explore the lived experiences of British Paralympic swimmers with respect to the personal and professional support available, perceived use of the support network and the influence it has on well-being and performance.

Methods and analysis A hermeneutic phenomenological study will be undertaken using a subtle-realist paradigmatic view. A purposive sample of British Paralympic swimmers will be recruited to enable exploration of social support experiences. In-depth semistructured interviews will explore participants' experiences of being an elite para-athlete, their support network, the social support available and how they perceive it relates to their well-being and performance. Strategies including reflexivity and member checking will be used to ensure trustworthiness. Data will be analysed following the Framework Method; a seven-stage process used for qualitative data analysis.

Ethics and dissemination This study has ethical approval (ERN_20-0344) granted by the University of Birmingham in April 2020. The findings of this study will be published in a peer-reviewed journal and disseminated to key stakeholders in elite para-sport to inform support services and improve athlete well-being and performance.

\section{INTRODUCTION}

\section{Rationale}

The Paralympic Games is a quadrennial global multisport event for athletes with impairments, referred to here as para-athletes. ${ }^{12}$ Since inception in 1960, the number of paraathletes competing at the Paralympic Games has increased 10-fold, with $>4300$ participants at Rio 2016. ${ }^{3}$ The 'Paralympic Movement', credited in part to the International
Strengths and limitations of this study

- This study will be the first to explore the experiences of social support in Paralympic swimmers, therefore, the findings produced will be novel.

- Rigorous methods will be employed at all stages of the study including reflexivity, member checking and pilot and cognitive interviews, increasing the credibility of the findings.

- This study will focus only on British Paralympic swimmers so the results may not be generalisable or transferable to other Paralympic sports or countries.

Paralympic Committee (IPC), has led to an increase in sporting opportunities for individuals with disabilities and raised the profile of elite para-sport. ${ }^{4-6}$ Para-athletes are classified for their sport based on eligible impairments, minimum disability criteria and sport classes, ${ }^{7}$ ensuring that the impact of impairment is minimised and that sporting excellence determines the success of an individual or team. ${ }^{8}$ The increase in para-sport awareness is concurrent with an increase in Paralympic Sport funding, with UK Sport investing almost $£ 75$ million into the Tokyo 2020 Paralympic Games 4-year cycle compared with $£ 10$ million for the Sydney Paralympic Games 20 years ago. ${ }^{9} 10$

Swimming has been featured at every Paralympic Games since inception. ${ }^{11}$ Paraswimmers are classified into three impairment groups: physical (S1-S10), visual (S11-S13) and intellectual (S14); with a lower number corresponding to a greater impairment and greater impact on performance. ${ }^{11}$ Second only to para-athletics, almost 600 swimmers competed at Rio 2016. ${ }^{12}$ British para-swimmers have achieved considerable success, finishing third in the medal table at Rio $2016^{12}$ and are in receipt of significant funding from UK Sport-just under £11 million for the most recent Paralympic cycle. ${ }^{13}$ 
Research in disability sport has emerged over the past decade with several studies investigating the benefits, ${ }^{14-17}$ barriers, ${ }^{15-22}$ facilitators ${ }^{19-23}$ and motivations ${ }^{2425}$ for sport participation across a range of ages, impairments and performance levels. Specifically in Paralympians, studies have examined athletic identity, ${ }^{26-28}$ retirement, ${ }^{29}$ injury experiences $^{30}$ and the barriers and facilitators to participation in elite sport. ${ }^{20}$ However, there is a paucity of evidence exploring the role of social support and the support network for Paralympians.

Four dimensions of support have been proposed comprising emotional, esteem, informational and tangible support; demonstrating the importance of different forms of support in elite sport. ${ }^{31}$

Sources of support have increased considerably over the years, from family, friends and coaches, ${ }^{32-34}$ to a whole contingency of support staff including nutritionists, physiotherapists and psychologists. ${ }^{35-37}$ The value of a holistic approach to performance enhancement has been highlighted in Olympic athletes, consisting of mental preparation, nutrition education and strength training, as well as having the support of friends, family and national governing bodies. ${ }^{356}$ The relatively new introduction of performance lifestyle (PL) advisors to sports including para-swimming highlights the importance that is being placed on athlete well-being and personal development, as well as performance. ${ }^{38} 39$

Exploration of the lifestyle and psychological factors impacting performance found that both personal and professional support were considered important to athletic success in Olympic and Paralympic athletes. ${ }^{37}$ However, as only two para-athletes participated in this study, definitive conclusions to inform practice in Paralympic sport were not possible. In non-elite para-swimmers, the providers of social support and their perceived importance have been investigated quantitatively, yet no research has qualitatively explored the social support experiences of paraswimmers. ${ }^{33}$ Significant increases in para-sport funding and resources have occurred since this study, ${ }^{4-6910}$ therefore, it is of interest to explore in-depth social support in Paralympic swimmers.

This protocol outlines the methods of a phenomenological qualitative study, which will be the first to explore the lived experiences of Paralympic swimmers and their support network. It is anticipated that the findings from this study will provide a deeper understanding of this phenomenon, enabling better support for Paralympic athletes, ultimately improving well-being and maximising performance outcomes.

\section{Aim}

To explore the lived experiences of social support in Paralympic swimmers.

\section{Objectives}

1. To determine the members of the Paralympic swimmers' support network and examine the personal and professional support available.
2. To explore the Paralympic swimmers' perceptions of the use of the support network and available support.

3. To explore the influence this support has on Paralympic swimmer well-being and performance.

\section{METHODS AND ANALYSIS}

\section{Theoretical framework and study design}

The philosophical position underpinning this qualitative study is that of subtle realism. This is consistent with a realist ontology (the belief that a world exists independently from our knowledge of it $)^{40}$ and subjectivist epistemology (knowledge is subjective, and the knower and the known are interdependent). ${ }^{41}$ Subtle realism considers that multiple non-contradictory and equally valid explanations exist for the same phenomena, and assumes that knowledge is based on assumptions and is a human construction. ${ }^{42}$ This framework rejects the view that beliefs are known with certainty, and claims there is not a single way of knowing. ${ }^{43}$

A hermeneutic phenomenological approach employing framework analysis is in keeping with these beliefs. Hermeneutic phenomenology was first proposed by Heidegger in 1927, and later developed further by Gadamer and van Manen. ${ }^{44}{ }^{45}$ Finlay ${ }^{46}$ states that this approach aims to 'evoke lived experience through the explicit involvement of interpretation' (pg. 110) and involves the researcher participating in the 'hermeneutic circle' between the whole text and parts of the text. ${ }^{445}$ This methodology is appropriate for this study as it aims to understand and interpret phenomena from the individual's perspective, allowing an insight into the experiences of British Paralympic swimmers. ${ }^{46}$ The methodology was informed by, and the study will be reported in accordance with, the Consolidated Criteria for Reporting Qualitative research (COREQ) to enable comprehensive reporting of this study. ${ }^{47}$

\section{Participant inclusion criteria}

Participants will be para-swimmers who have competed at a Paralympic Games for Great Britain and are actively training and competing. They will have an impairment that complies with the IPC classification code. ${ }^{48}$ Participants will be able to communicate fluently in English and able and willing to give informed consent. There will be no age limit and both males and females will be included.

\section{Participant recruitment}

Phenomenology promotes the recruitment of participants from a homogeneous population who have had experience with the phenomenon, but who vary in characteristics and their personal experience. ${ }^{49}$ Therefore, purposive sampling will be used to identify potential participants based on age, gender, impairment and Paralympic experience, because this will enable the provision of rich, diverse data pertinent to the research objectives of social support experiences. ${ }^{50}$ Athletes will be approached and contacted via email and social media to determine interest in participation. Contacts in the 
authors' sporting networks will be approached to aid the recruitment of participants.

Sample size will be informed by information power as proposed by Malterud et al. ${ }^{51}$ This is a tool used in qualitative interview studies and is based on the concept that the larger the information power of a sample, the fewer participants are needed. ${ }^{51}$ The study aim is narrow, there is high sample specificity and the case analysis strategy suggests few participants are required. This study will follow the theoretical framework and four dimensions of social support including emotional, esteem, informational and tangible support, proposed by Rees and Hardy. ${ }^{31}$ The lead researcher (BA) has strong communication skills, competed nationally as a swimmer for 10 years and has experience training alongside elite paraswimmers. The research team also comprised expert qualitative researchers (NRH, ABR and AS) and a parasport practitioner $(\mathrm{PM})$. Based on these dimensions of information power, it is estimated that the sample size will be approximately eight participants. ${ }^{51}$

\section{Study setting}

Video calls will be conducted from the researcher's and participants' homes. Given the current restrictions on contact and social distancing as a result of COVID-19 the ethics committee support the use of an electronic video platform to conduct interviews.

\section{Data collection}

The planned start date for the study is May 2020 and the planned end date is August 2020. Data will be collected via semistructured interviews conducted by the lead researcher, a female MSc student, enabling participants to give a detailed first-person account of their experiences. ${ }^{44}$ One interview will be conducted with each participant via video call between May and July 2020 at a date and time convenient for the participant. This method of online face-to-face interviewing is often more convenient for participants while still enabling observation of verbal and non-verbal cues. ${ }^{52}$ It has also been suggested that there is little difference in the data quality between online and face-to-face interviews, further supporting the use of video interviews. ${ }^{53}$ At least 24 hours prior to participation, informed consent will be obtained for the interview and for audiorecording. As is recommended in phenomenological studies, the interviewer will endeavour to create a relaxed, comfortable environment for the participant and engage them in conversation prior to the interview. This will facilitate the development of rapport and make the participant feel more comfortable and more likely to give a rich account. ${ }^{54}$ Prior to the interview the participant will be reminded of the purpose of the research, that participation is voluntary and that they can with suspend the interview at any point.

A topic guide (online supplemental file 1) informed by existing evidence ${ }^{31} 37$ and conversations with elite para-sport practitioners in PL and Physiotherapy has been created to guide the interviews. Questions are open-ended and participants will be invited to discuss any concepts or thoughts they feel are relevant. The guide includes introductory questions on sporting background, classification, achievements, Paralympic games and overall experience of being a Paralympic swimmer. The main section comprises questions on the experiences of emotional, esteem, informational and tangible support, structural support and social networks. Prompts will be given if necessary and clarification sought if required.

The interviews are estimated to last between 45 and $60 \mathrm{~min}$ and will be audiorecorded and transcribed verbatim. Cognitive and pilot interviews will be undertaken with approximately two Paralympians from individual sports to assess the understanding and interpretation of the interview questions and refine interview techniques. ${ }^{556}$ Based on feedback amendments will be to the topic guide if necessary. Field notes will be taken throughout the interviews to provide contextual details and to note non-verbal expressions, aiding data analysis and interpretation. ${ }^{57}{ }^{58} \mathrm{~A}$ reflexive diary will be used to record the details of the nature and origin of any interpretations which emerge through the interview process; aiding trustworthiness. ${ }^{45} \mathrm{~A}$ copy of the transcript will be emailed to each participant to enable member checking. Each participant will be able to add any further reflections or make alterations, ensuring an accurate representation of their perspectives and experiences, adding to the transparency of the research. ${ }^{579}$

\section{Data analysis}

The lead researcher will be responsible for analysing the interview data because of her experience in competitive swimming and training alongside para-swimmers. In hermeneutic phenomenology, the researchers' biases, assumptions and experiences are essential to data analysis and interpretation, ${ }^{44-46}$ supporting the reflexive diary. Participant quotations will be used to illustrate key findings, making the results more credible ${ }^{60}$ Analysis will be carried out immediately after interviews and after approximately 2-3 interviews it will be determined whether the topic guide needs adapting depending on responses to provide more focus.

The Framework Method (a seven-stage process for qualitative data management and analysis) is an established approach commonly used to analyse qualitative data; hence it is appropriate in examining and understanding lived experience of phenomena. ${ }^{61}$ The method highlights the importance of the involvement of individuals with experience in qualitative research, therefore, experienced qualitative researchers will be involved at every stage of analysis (NRH, ABR and AS) ${ }^{61}$ The sevenstage procedure based on that proposed by Gale $e t a t^{61}$ will be adopted to analyse the data (figure 1). It is possible that the analysis approach will be subject to change due to the inductive nature of qualitative work and the multiple types of analysis, which involves the immersion of text and initial open coding. Following these stages, the themes or focus may be towards other concepts or phenomena 


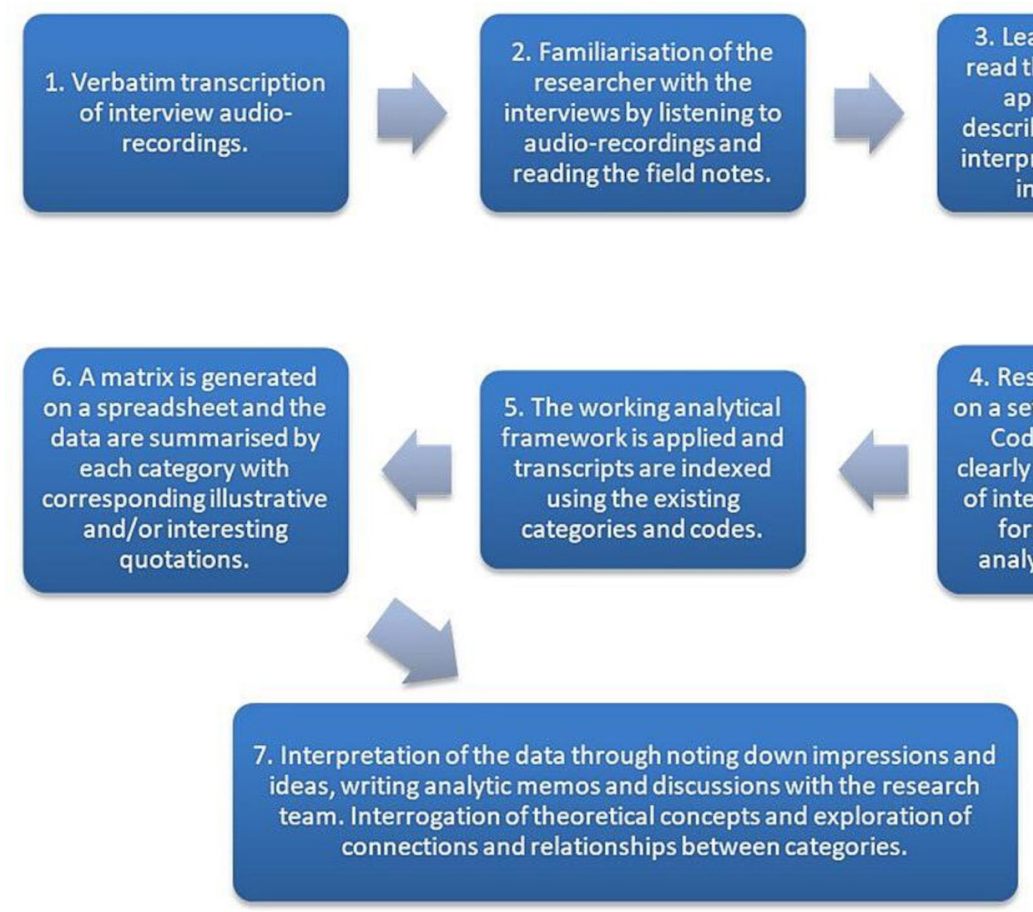

Lead researcher will

read the transcripts and applya code that

describes what has been

interpreted as important in the passage.

Research team agree

a set of codes to apply. Codes grouped into

learly defined categories

finterrelated concepts,

forming a working

nalytical framework

Figure 1 The framework method.

due to the unique environment and sample that is being considered.

\section{PATIENT AND PUBLIC INVOLVEMENT}

This qualitative study and protocol have been informed through contact with elite para-athletes and practitioners working in elite para-sport. Elite para-athletes will contribute to the topic guide content and structure through cognitive interviews. Key stakeholders in elite para-sport and para-swimming may be contacted for their contribution and insight to help aid analysis and interpretation of results.

\section{ETHICS AND DISSEMINATION}

Institutional ethical approval was obtained from the University of Birmingham in April 2020 (ERN_20-0344). Participants will sign a consent form and receive a participant information sheet prior to the interview. They will have the right to withdraw from the study prior to the interview and within 4 weeks after the interview. There are minimal risks associated with this study. In the unlikely event that a participant feels distressed or the interviewer is concerned for the participant's well-being, the interview will be suspended and the participant informed of relevant support services. Participant data will be stored confidentially for 10 years on password-protected computers that can only be accessed by the researchers, and in accordance with General Data Protection Regulation, the Data Protection Act 2018 and University of Birmingham's research governance frameworks. When presenting the study findings, pseudonyms will be used to protect the participants' identities due to the relatively small population of current British Paralympic swimmers. Participation in the study will be entirely voluntary and no incentives will be offered. Any protocol deviations will be documented. The findings from this research will be disseminated to key stakeholders in elite para-sport and communicated to participants as a summary report.

\section{DISCUSSION}

This protocol outlines the rationale and methodology of a qualitative study, which will investigate the lived experiences of social support in British Paralympic swimmers. The lead researcher has experience of competitive swimming and training with elite para-swimmers, therefore, it is anticipated that there may be some element of researcher bias. One limitation to this study is that the researcher does not have experience in conducting interviews however; pilot interviews will enable the practice and refinement of interview techniques. Also, limiting the sample to British Paralympic swimmers may reduce the generalisability and applicability of the results. The results from this qualitative study will serve to inform researchers about the lived experiences of Paralympic swimmers. It is anticipated that the findings from this study will give a deeper understanding of what it is like to be an elite-para athlete and will facilitate increased support for para-athletes in order to maximise their wellbeing and performance.

Twitter Andrew Soundy @Andy_Soundy, Alison Rushton @abrushton and Nicola R Heneghan @HeneghanNicola 
Contributors All authors devised the focus of this review protocol. BA is a MSc by Research student, NRH is the lead supervisor, AR and AS are co-supervisors and PM is an expert in para sport. BA drafted the initial protocol manuscript with lead and co-supervisors providing guidance on methodological decisions and proposed analyses. All authors have contributed subject-specific and/or methodological expertise. BA will recruit participants and collect data, supported by supervisors. All authors will contribute to data interpretation, conclusions and dissemination. All authors have read, contributed to and agreed to the final manuscript. NRH is the guarantor of the study.

Funding The authors have not declared a specific grant for this research from any funding agency in the public, commercial or not-for-profit sectors.

\section{Competing interests None declared.}

Patient and public involvement Patients and/or the public were involved in the design, or conduct, or reporting, or dissemination plans of this research. Refer to the Methods section for further details.

Patient consent for publication Not required.

Provenance and peer review Not commissioned; externally peer reviewed.

Open access This is an open access article distributed in accordance with the Creative Commons Attribution Non Commercial (CC BY-NC 4.0) license, which permits others to distribute, remix, adapt, build upon this work non-commercially, and license their derivative works on different terms, provided the original work is properly cited, appropriate credit is given, any changes made indicated, and the use is non-commercial. See: http://creativecommons.org/licenses/by-nc/4.0/.

\section{ORCID iDs}

Andrew Soundy http://orcid.org/0000-0002-5118-5872

Alison Rushton http://orcid.org/0000-0001-8114-7669

Nicola R Heneghan http://orcid.org/0000-0001-7599-3674

\section{REFERENCES}

$1 \mathrm{Kim} \mathrm{KT}$, Lee S, Oh E-S. Athletes with disabilities in the Paralympic games: a framing analysis of television news. Manag Sport Leis 2017;22:255-75

2 McPherson G, O'Donnell H, McGillivray D, et al. Elite athletes or superstars? media representation of para-athletes at the Glasgow 2014 Commonwealth games. Disabil Soc 2016;31:659-75.

3 International Paralympic Committee. Summer paralympic games overview. Available: https://www.paralympic.org/paralympic-games/ summer-overview [Accessed 3 Jan 2020].

4 Blauwet C, Willick SE. The Paralympic movement: using sports to promote health, disability rights, and social integration for athletes with disabilities. Pm R 2012;4:851-6.

5 Thomas N, Disability SA. Disability, sport and society: an introduction. London: Routledge, 2008.

6 International Paralympic Committee. History of the Paralympic movement. Available: https://www.paralympic.org/ipc/history [Accessed 3 Jan 2020].

7 International Paralympic Committee. Classification explained. Available: https://www.paralympic.org/classification [Accessed 3 Jan 2020].

8 International Paralympic Committee. IPC athlete classification code. Available: https://www.paralympic.org/sites/default/files/document/ 170704160235698_2015_12_17\%2BClassification\%2BCode_ FINAL2 0.pdf [Accessed 3 Jan 2020].

9 UK Sport. Current funding figures. Available: https://www.uksport. gov.uk/our-work/investing-in-sport/current-funding-figures [Accessed 3 Jan 2020].

10 UK Sport. Historical funding figures. Available: https://www.uksport. gov.uk/our-work/investing-in-sport/historical-funding-figures [Accessed 3 Jan 2020].

11 Espanol. World para swimming. history of para swimming. Available: https://www.paralympic.org/swimming/about [Accessed 3 Apr 2020]

12 Espanol. Rio 2016 Paralympic Games. results archive Rio, 2016. Available: https://www.paralympic.org/rio-2016/results/swimming [Accessed 3 Apr 2020].

13 UK Sport. Para-swimming. Available: https://www.uksport.gov.uk/ sports/paralympic/para-swimming [Accessed 3 Apr 2020].

14 Hudson NA, Mrozik JH, White R, et al. Community football teams for people with intellectual disabilities in secure settings: "They take you off the ward, it was like a nice day, and then you get like medals at the end". J Appl Res Intellect Disabil 2018;31:213-25.
15 Darcy S, Dowse L. In search of a level playing field - the constraints and benefits of sport participation for people with intellectual disability. Disabil Soc 2013;28:393-407.

16 Bowers K, Corby D, Lambert V, et al. People with intellectual disability and their families' perspectives of special Olympics ireland: qualitative findings from the SOPHIE study. J Intellect Disabil 2016;20:354-70.

17 Barfield JP, Malone LA. Perceived exercise benefits and barriers among power wheelchair soccer players. J Rehabil Res Dev 2013;50:231-8.

18 Wilson NC, Khoo S. Benefits and barriers to sports participation for athletes with disabilities: the case of Malaysia. Disabil Soc 2013;28:1132-45.

19 Jaarsma EA, Dekker R, Koopmans SA, et al. Barriers to and facilitators of sports participation in people with visual impairments. Adapt Phys Activ Q 2014;31:240-64.

20 Jaarsma EA, Geertzen JHB, de Jong R, et al. Barriers and facilitators of sports in Dutch Paralympic athletes: an explorative study. Scand J Med Sci Sports 2014;24:830-6.

21 Jaarsma EA, Dijkstra PU, de Blécourt ACE, et al. Barriers and facilitators of sports in children with physical disabilities: a mixedmethod study. Disabil Rehabil 2015;37:1617-25.

22 Hutzler Y, Bergman U. Facilitators and barriers to participation while pursuing an athletic career. Ther Recreation J2011;45:1-6.

23 Bragaru M, van Wilgen CP, Geertzen JHB, et al. Barriers and facilitators of participation in sports: a qualitative study on Dutch individuals with lower limb amputation. PLoS One 2013;8:e59881.

24 Farrell RJ, Crocker PRE, McDonough $\mathrm{MH}$, et al. The driving force: motivation in special Olympians. Adapt Phys Activ $Q$ 2004;21:153-66.

25 Kirkby RJ. Wheelchair netball: motives and attitudes of competitors with and without disabilities. Aust Psychol 1995;30:109-12.

26 Swartz L, Bantjes J, Knight B, et al. "They don't understand that we also exist": South African participants in competitive disability sport and the politics of identity. Disabil Rehabil 2018;40:35-41.

27 Pack S, Kelly S, Arvinen-Barrow M. "I think I became a swimmer rather than just someone with a disability swimming up and down:" paralympic athletes perceptions of self and identity development. Disabil Rehabil 2017;39:2063-70.

28 de Haan D, Sotiriadou P, Henry I. The lived experience of sexintegrated sport and the construction of athlete identity within the Olympic and Paralympic Equestrian disciplines. Sport Soc 2016;19:1249-66.

29 Bundon A, Ashfield A, Smith B, et al. Struggling to stay and struggling to leave: the experiences of elite para-athletes at the end of their sport careers. Psychol Sport Exerc 2018;37:296-305.

30 Fagher K, Forsberg A, Jacobsson J, et al. Paralympic athletes' perceptions of their experiences of sports-related injuries, risk factors and preventive possibilities. Eur J Sport Sci 2016;16:1240-9.

31 Rees T, Hardy L. An investigation of the social support experiences of high-level sports performers. Sport Psychologist 2000;14:327-47.

32 Rosenfeld LB, Richman JM, Hardy CJ. Examining social support networks among athletes: description and relationship to stress. Sport Psychol 1989;3:23-33.

33 Martin JJ, Mushett CA. Social support mechanisms among athletes with disabilities. Adapt Phys Activ Q 1996;13:74-83.

34 Barefield S, McCallister S. Social support in the athletic training room: athletes' expectations of staff and student athletic trainers. $J$ Athl Train 1997;32:333.

35 Gould D, Guinan D, Greenleaf C, et al. Factors affecting Olympic performance: perceptions of athletes and coaches from more and less successful teams. Sport Psychol 1999;13:371-94.

36 Greenleaf C, Gould D, Dieffenbach K. Factors influencing Olympic performance: interviews with Atlanta and Negano us Olympians. $J$ Appl Sport Psychol 2001;13:154-84.

37 Burns L, Weissensteiner JR, Cohen M. Lifestyles and mindsets of Olympic, Paralympic and world champions: is an integrated approach the key to elite performance? Br J Sports Med 2019;53:818-24.

38 English Institute of Sport. Performance lifestyle. Available: https:// www.eis2win.co.uk/service/performance-lifestyle/[Accessed 3 Apr 2020].

39 Ashfield A, Harrison J, Giles S. Performance lifestyle in Olympic and Paralympic sport. In: Brady A, Grenville-Cleave B, eds. Positive psychology in sport and physical activity. An introduction. London: Routledge, 2017.

40 Sayer A. Realism and social science, 2000. Available: https://pdfs. semanticscholar.org/a852/2bad59dd5b62b2b3b0bd4b116215 61fa0327.pdf [Accessed 4 Apr 2020].

41 Sparkes AC, Smith B. Qualitative research methods in sport, exercise and health: from process to product, 2014. Available: 
https://ebookcentral.proquest.com/lib/bham/reader.action?doclD= 1480779\&ppg=10 [Accessed 4 Apr 2020].

42 Duncan EAS, Nicol MM. Subtle realism and occupational therapy: an alternative approach to knowledge generation and evaluation. $\mathrm{Br} J$ Occup Ther 2004;67:453-6.

43 Hammersley M. Ethnography and realism. In: Huberman AM, Miles MB, eds. Qualitative researchers companion. Thousand Oaks, USA: Sage, 2002: 65-80.

44 Sloan A, Bowe B. Phenomenology and hermeneutic phenomenology: the philosophy, the methodologies, and using hermeneutic phenomenology to investigate lecturers' experiences of curriculum design. Qual Quant 2014;48:1291-303.

45 Laverty SM. Hermeneutic phenomenology and phenomenology: a comparison of historical and methodological considerations. Int J Qual Methods 2003;2:21-35.

46 Finlay L. Phenomenology for therapists: researching the lived world, 2011. Available: https://ebookcentral.proquest.com/lib/bham/reader. action?docID=819252 [Accessed 4 Apr 2020].

47 Tong A, Sainsbury P, Craig J. Consolidated criteria for reporting qualitative research (COREQ): a 32-item checklist for interviews and focus groups. Int J Qual Health Care 2007;19:349-57.

48 International Paralympic Committee. Explanatory guide to paralympic classification. Available: https://www.paralympic.org/ sites/default/files/document/150915170806821_2015_09_15\% 2BExplanatory\%2Bguide\%2BClassification_summer\%2BFINAL\% 2B_5.pdf [Accessed 4 Jan 2020].

49 Moser A, Korstjens I. Series: practical guidance to qualitative research. Part 3: sampling, data collection and analysis. Eur J Gen Pract 2018;24:9-18.

50 Giacomini MK, Cook DJ. Users' guides to the medical literature: XXIII. qualitative research in health care A. are the results of the study valid? evidence-based medicine Working group. JAMA 2000;284:357-62.

51 Malterud K, Siersma VD, Guassora AD. Sample size in qualitative interview studies: guided by information power. Qual Health Res 2016;26:1753-60.

52 Janghorban R, Latifnejad Roudsari R, Taghipour A. Skype interviewing: the new generation of online synchronous interview in qualitative research. Int J Qual Stud Health Well-being 2014;9:24152.

53 Weller S. Using Internet video calls in qualitative (longitudinal) interviews: some implications for rapport. Int J Soc Res Methodol 2017;20:613-25.

54 Smith JA, Flowers P, Larkin M, et al. Theory, method and research, 2009.

55 Griffee DT. Research tips: interview data collection. J Dev Educ 2005;28:36-7.

56 Knafl K, Deatrick J, Gallo A, et al. The analysis and interpretation of cognitive interviews for instrument development. Res Nurs Health 2007;30:224-34.

57 Fossey E, Harvey C, McDermott F, et al. Understanding and evaluating qualitative research. Aust N Z J Psychiatry 2002;36:717-32.

58 Emerson RM, Fretz RI, Shaw LL. Writing ethnographic fieldnotes. Chicago: University of Chicago Press, 2011.

59 Thomas DR. Feedback from research participants: are member checks useful in qualitative research? Qual Res Psychol 2017; 14:23-41.

60 Côté L, Turgeon J. Appraising qualitative research articles in medicine and medical education. Med Teach 2005;27:71-5.

61 Gale NK, Heath G, Cameron E, et al. Using the framework method for the analysis of qualitative data in multi-disciplinary health research. BMC Med Res Methodol 2013;13:117. 